\title{
Influencia de los Perfiles Docentes en el Uso y Preferencia de Dinámicas de Trabajo en Grupo con Alumnos Universitarios
}

\author{
Juan A. Marin-Garcia ${ }^{(1)}$ y Andrea Conchado-Peiró( ${ }^{(2)}$ \\ (1) Universidad Politécnica de Valencia, ROGLE- Departamento de Organización de Empresas \\ (DOE), Camino de Vera s/n, 46022 Valencia-España (e-mail: jamarin@omp.upv.es) \\ (2) Universidad Politécnica de Valencia, Departamento de Estadística, Investigación Operativa \\ Aplicadas y Calidad (DEIOAC), Camino de Vera s/n, 46022 Valencia-España \\ (e-mail: anconpei@upvnet.upv.es)
}

Recibido Mar. 16, 2012; Aceptado Abr. 13, 2012; Versión final recibida May. 04, 2012

\begin{abstract}
Resumen
El objetivo del presente trabajo fue analizar el uso y las preferencias de uso entre el profesorado universitario sobre nueve dinámicas de trabajo en grupo. Para ello se realizó una encuesta a una muestra de 193 profesores de universidades españolas. El análisis de datos mediante análisis de correspondencias múltiples permitió identificar tres grupos de profesores en cuanto al uso de estas técnicas y otros dos adicionales relativos a las preferencias de uso. Dichos perfiles fueron caracterizados por medio de la información sobre el perfil profesional de los profesores. Concretamente los factores más significativos resultaron ser el área de conocimiento, la experiencia docente, y los conocimientos y opinión que se posee de las metodologías activas.
\end{abstract}

\section{Influence of Instructor's Profiles on the Use and Preference of Teamwork Dynamics with University Students}

\begin{abstract}
The aim of this work was to analyze the use and usage preferences among university instructors about nine teamwork dynamics. This survey was conducted on a sample of 193 Spanish university professors. Data analysis by multiple correspondence analysis identified three groups of teachers in the use of these techniques and an additional two others concerning usage preferences. These profiles were characterized by means of information about the professional profile of the instructors. Specifically, the most significant factors were found to be the area of knowledge, teaching experience, and knowledge and opinion that the teacher has on active methodologies.
\end{abstract}

Keywords: active methodology, higher education, team, group, active learning 


\section{INTRODUCCIÓN}

Desde diversas fuentes se han manifestado las ventajas que proporciona el plantear una metodología basada en trabajo en grupo con alumnos universitarios (Anson et al., 2003; Kalliath y Laiken, 2006; Marin-Garcia et al., 2008; Watts et al., 2006). Por un lado, permite formar a los alumnos en las competencias profesionales de muchas titulaciones universitarias experimentando y adquiriendo las habilidades que necesitarán en sus futuros puestos de trabajo (Marin-Garcia et al., 2008; Marin-Garcia et al., 2009; Marin-Garcia et al., 2010; Raposo Rivas y Martínez Figueira, 2011). Algunas de estas habilidades son: comunicación interpersonal (Brewer y Mendelson, 2003; Jenkins y Lackey, 2005), trabajo en equipo (Fruchter, 2001; Kalliath y Laiken, 2006; Michaelson, 2003; Young y Henquinet, 2000), solución de problemas en grupo (Brewer y Mendelson, 2003; Jenkins y Lackey, 2005), liderazgo (Jenkins y Lackey, 2005; Sheppard et al., 2004), negociación (Michaelson, 2003; Sheppard et al., 2004) y gestión del tiempo (Jenkins y Lackey, 2005).

Por otro lado, el trabajo en grupo proporciona un aprendizaje más profundo y significativo de los contenidos, cuando es utilizado dentro de un contexto de metodologías activas (Young y Henquinet, 2000). Además, se han demostrado efectos positivos en el rendimiento académico de los estudiantes, la motivación y su actitud hacia el aprendizaje (Kalliath y Laiken, 2006; Martínez Gómez y Marin-Garcia, 2009; Michaelson, 2003; Watts et al., 2006). Algunas de estas ventajas han sido resaltadas también por los estudiantes, que consideran las actividades de grupo como más interesantes, divertidas y facilitadoras de aprendizaje que la docencia tradicional (Watts et al., 2006).

Por todo ello, el trabajo en grupo ha sido un aspecto importante en la docencia universitaria (O'Doherty, 2005). Sin embargo, el trabajo en grupo también genera problemas. Por ejemplo, en determinados contextos existe resistencia por parte de los estudiantes, que no están habituados a esta forma de trabajar y se sienten desorientados (Holtham et al., 2006), o que consideran que estas actividades les obligan a invertir mucho tiempo (Holtham et al., 2006; Marin-Garcia y Lloret, 2008; Palou y Montaño, 2008). En otros casos, la resistencia puede provenir de los profesores. Entre las justificaciones más habituales para no incorporar actividades en grupo a la docencia, podemos encontrar que las asignaturas no disponen de tiempo de docencia para perderlo en actividades de grupo que son lentas e impiden que se pueda completar el temario; el profesor no sabe muy bien cómo organizar las actividades de grupo o no dispone de tiempo para prepararlas (Holtham et al., 2006). Además, los profesores temen perder el control de la clase (Michaelson, 2003)

En este sentido el objetivo del presente trabajo es aportar más datos al respecto respondiendo a la siguiente pregunta de investigación: ¿existen diferencias en el grado de uso de las dinámicas de grupos entre profesores en función del conocimiento sobre el tema, antigüedad dando clases, responsabilidad en la asignatura, área de conocimiento, estabilización o punto de vista sobre las metodologías activas?

\section{TRABAJO EN GRUPO DE LOS ESTUDIANTES UNIVERSITARIOS}

Con el término grupo nos referimos a un pequeño número de personas interdependientes con habilidades complementarias, que interactúan para adquirir conocimientos, habilidades o actitudes y producir resultados en común (Brewer y Mendelson, 2003; Raposo Rivas y Martínez Figueira, 2011; Watts et al., 2006). Existen varias técnicas que nos permiten hacer trabajar en grupo a nuestros alumnos (Auster y Wylie, 2006; Fabra, 1994; Lloret y Marin-Garcia, 2008; Marin-Garcia et al., 2008), entre ellas destacamos las siguientes: Dramatizaciones (JR): representar en un espacio concreto la acción dramática previamente definida con los personajes escogidos. Las personas que intervienen interactúan libremente en el marco y la situación elegidos y cada uno va adecuando su papel al de los demás. Pecera (PC): consiste en formar dos círculos concéntricos de personas, uno de los cuales (el que está en el interior) discute o actúa sobre un tema, mientras el otro grupo observa. Los observadores pueden tener o no unos criterios de observación preestablecidos. Puzzle (PZ): consiste en dividir un grupo numeroso en subgrupos (por ejemplo un grupo de treinta personas en seis grupos de cinco componentes). Estos subgrupos 
interaccionan durante un tiempo para realizar alguna tarea. Finalizado el tiempo, o bien se elige un portavoz que expone ante los demás grupos las conclusiones del subgrupo, o se numeran los componentes y se crean cinco nuevos grupos de seis componentes (todos los 1 juntos, etc.). Dinamizadores (DN): ejercicios breves y desenfadados que pretenden liberar la creatividad de los participantes, fomentar un clima adecuado en el aula y facilitar el desarrollo de las otras dinámicas. Tormenta de ideas (BS): una técnica para generar un amplio número de ideas. Los participantes van expresando sus ideas a medida que se van produciendo, sin preocuparse de su aplicabilidad o de otro tipo de filtros mentales. Se puede llevar a cabo con múltiples variantes: los componentes aportan sus ideas de viva voz sin haber establecido ningún turno; de viva voz, pero siguiendo un turno; usando notas de papel para recopilar las ideas (lo que permite reorganizarlas con más facilidad). Esta última variante se suele denominar "grupo nominal". Voto múltiple (VM): consiste en puntuar una lista de ideas, bien dando puntos del 1 al 10 a cada idea o restringiendo el número de votos que puede emitir cada persona (por ejemplo, votar sólo las 4 ideas más importantes de la lista). Se ordenan las ideas en función del número de votos y el grupo discute y resume los resultados. Se puede usar en combinación de otras técnicas como la tormenta de ideas. Murmullos (MM): permitir a los estudiantes que dialoguen en parejas un breve espacio de tiempo (3-5 minutos) para expresar sus dudas o sus opiniones o resumir lo que se ha explicado o resaltar lo que les ha parecido más interesante. Una alternativa de presentación de esta dinámica son los "círculos concéntricos" Bola de nieve (BN): consiste en empezar con trabajo en parejas. Luego se juntan dos parejas para formar grupos de cuatro alumnos que profundizan o integran el trabajo de las parejas. Luego se juntan dos grupos de cuatro para integrar las opiniones de los 8 nuevos componentes, etc.

En la literatura previa se han comentado algunas variables que pueden tener incidencia en el grado de uso de las dinámicas de grupo, bien porque condicionan su utilización o bien porque complican el uso y hace menos probable que los profesores estén dispuestos a usar dinámicas de grupo en tales circunstancias. Uno de los temas recurrentes tiene como centro los contenidos de la asignatura. En este sentido, la opinión mayoritaria considera que las dinámicas de grupo se pueden usar prácticamente en cualquier disciplina. Sin embargo, una de las manifestaciones frecuentes de algunos profesores es "esto va bien para los de letras, pero no se puede aplicar en mi asignatura" (Lloret y Marin-Garcia, 2007).

También se ha comentado que el modo de pensar (creencias) de los profesores, y su formación, puede condicionar, en mayor o menor medida, su actuación en el aula (Contreras Palma, 2008). En este sentido cuanto mayor sea la preparación o experiencia previa con metodologías activas 0 una actitud favorable del profesor hacia las mismas, más probable es que los profesores usen dinámicas de grupo en sus clases.

Por otra parte, la capacidad de decisión en la asignatura (por ejemplo, el hecho de ser responsable de la misma) puede facilitar la incorporación de nuevas metodologías alternativas a la docencia tradicional. Relacionado con esto, hay cierta tendencia a considerar que los años en el puesto o la estabilidad pueden generar cierta docencia tradicional o inmovilista. Pero, al mismo tiempo, la seguridad en el puesto y no estar sometido a la presión de "tener que gustar" para garantizar una promoción, podrían ser un catalizador para la innovación ya que no peligra el puesto de trabajo aunque se tomen decisiones arriesgadas o se fracase en los experimentos docentes. No obstante, no podemos obviar que existen posibles relaciones cruzadas entre las variables incluidas en el estudio. Cuantos más años se lleve impartiendo docencia, es más probable que el profesor pueda ser responsable de asignaturas o que tenga una figura contractual estabilizada como funcionario. También es posible que los años de docencia contribuyan a que haya aprendido a utilizar dinámicas de grupo y, eso reforzaría la predisposición a usarlas. Puesto que no hay una posición concluyente al respecto, nuestra investigación puede ayudar a clarificar esta situación.

\section{METODOLOGÍA}

Con el fin de conocer el grado de uso y las preferencias de los profesores universitarios se realizó una encuesta dirigida específicamente a este colectivo. El instrumento fundamental de esta 
encuesta fue un cuestionario estructurado en dos secciones. La primera sección contenía diversas preguntas relativas al uso y preferencias por las siguientes dinámicas de grupo: Juego de rol (JR), Pecera (PC), Puzzle (PZ), Dinamizadores (DN), Grupos nominales (GN), Tormenta de ideas o Brainstorming (BS), Voto múltiple (VM), Murmullos (MM) y Bola de nieve (BN). La segunda sección planteaba cuestiones relacionadas con el perfil profesional de los docentes. Todas las preguntas del cuestionario fueron formuladas de forma nominal o cualitativa, de forma que el entrevistado disponía de un conjunto cerrado de opciones exclusivas entre las que escoger. Concretamente dichas opciones incluían: No se puede aplicar a mi asignatura, No la he usado, La he usado y me gusta y La he usado y no me gusta.

Este cuestionario fue cumplimentado por 193 profesores universitarios pertenecientes a 3 universidades públicas de España (Universidad Politécnica de Valencia, Universidad de Murcia y Universidad de la Coruña). A partir de esta muestra no se pretende extraer conclusiones que puedan será inferidas sobre la población del profesorado universitario a nivel nacional, sino describir las preferencias de los profesores de las universidades seleccionadas en cuanto al uso de dinámicas de trabajo en grupo. La muestra de conveniencia se obtuvo por el procedimiento de bola de nieve, incorporando a todos los asistentes a talleres de formación de profesorado sobre el trabajo en grupo con alumnos universitarios. En total, un $51,6 \%$ de los encuestados fueron mujeres. Por áreas, la distribución fue Ciencias (13,8\%), Ciencias de la Vida y la Salud $(25,8 \%)$, Ciencias Tecnológicas (24,5\%) y Humanidades (35,8\%). El 64,2\% de los profesores incluidos en la muestra tienen al menos 6 años de experiencia en docencia universitaria y el porcentaje de funcionarios es del 38,1\%. Asimismo, el 74\% afirman tener una opinión favorable o muy favorable respecto al uso de dinámicas de trabajo en grupo, a pesar de que el $84 \%$ confiesa tener conocimientos sobre estas técnicas a nivel de principiante. Este último resultado, que en principio puede parecer una limitación en cuanto a la capacidad de los profesores entrevistados, constituye una característica generalizada en el ámbito universitario español. A pesar de que se están haciendo grandes esfuerzos a nivel institucional, en la mayoría de universidades españolas, para la formación para el empleo de las metodologías activas, todavía una amplia mayoría de profesores percibe ciertas carencias. La selección de profesores específicamente formados en esta competencia hubiera supuesto la introducción de un sesgo evidente en las valoraciones de los encuestados.

Así, se ha empleado análisis de correspondencias múltiples para identificar posibles agrupaciones en base al uso y preferencias de las dinámicas de trabajo en grupo expuestas anteriormente. El objetivo de esta técnica es la identificación de relaciones en un conjunto de variables cualitativas, cada una de las cuales consta de dos o más categorías, como ocurre en este caso. Esta técnica analiza las relaciones entre las distintas categorías de las variables, y aporta como resultado del análisis un diagrama bidimensional (Lizasoain y Joaristi, 2012). La posición de cada categoría de variable en este diagrama aporta información acerca de las relaciones que se establecen entre variables, ya que la proximidad indica relación o asociación entre categorías de variables, mientras que la lejanía o separación indica falta de relación o asociación (Greenacre, 2008). No obstante, la importancia del análisis no radica en la localización exacta de cada categoría en el diagrama, sino en la cercanía o lejanía entre categorías de variables. En este trabajo se han realizado dos análisis de correspondencias múltiples a partir de 2 agrupaciones de las categorías de respuesta planteadas para el uso y preferencia de las técnicas de trabajo en grupo. En el primero de ellos, referido exclusivamente al uso, se han considerado únicamente las categorías $L a$ he Usado (U), No la he Usado (NU) y No Aplica (NA). Puesto que se han seleccionado nueve dinámicas de trabajo en grupo, la primera representación bidimensional (Figura 1) muestra las proyecciones de 27 categorías de respuesta, logrando explicar de esta forma el 68\% de la variabilidad de los datos. En el segundo análisis se han incluido adicionalmente las categorías $L a$ he Usado y me Gusta (UG) y La he Usado y No me Gusta (UNG), que sustituyen conjuntamente a la anterior categoría La he Usado (U). De este modo, la Figura 2 presenta las proyecciones de 40 categorías de respuesta en un plano bidimensional, que explica el $54,5 \%$ de la varianza de los datos. En términos generales cabe señalar que no resulta apropiado el empleo de tasas de inercia como indicador para la evaluación global de la calidad de una representación obtenida mediante análisis de correspondencias múltiples. La principal razón es que la varianza bruta inicial no constituye, en general, una medida de referencia adecuada por lo que no es conveniente hacer 
referencia a tasas de inercia respecto a esta cantidad inicial, ya que resultan excesivamente pesimistas (Greenacre, 2008). Por otro lado se ha recurrido al empleo de elementos suplementarios como medio para introducir variables relativas al perfil del profesorado universitario y facilitar la interpretación del diagrama bidimensional (Lebart et al., 1995). Así, este análisis aporta la posibilidad de incrementar la información referida a los factores mediante variables que en principio no habían sido incluidas en la construcción de los ejes y permite conocer la posición de las variables suplementarias en el espacio previamente definido por las variables activas. En este trabajo la extracción de los factores se ha aplicado sobre el conjunto de las variables relativas al uso y preferencia por las distintas dinámicas de trabajo en grupo, y posteriormente se han proyectado en suplementario el área de estudio del departamento donde cada profesor imparte docencia.

\section{RESULTADOS Y DISCUSIÓN}

La Tabla 1 muestra los porcentajes de uso de las dinámicas de trabajo en grupo. En esta tabla se observa que las técnicas de uso mayoritario son la tormenta de ideas, el puzzle, los dinamizadores y los murmullos. Por el contrario, las técnicas menos usadas son la pecera, la bola de nieve y el voto múltiple. Sin embargo, su falta de uso no implica rechazo por parte del profesorado universitario. De hecho, el juego de rol y los grupos nominales, que se sitúan en posiciones intermedias en cuanto al uso, alcanzan los porcentajes más elevados en la categoría No aplica. Este resultado es indicativo de cierto rechazo hacia ambas técnicas por parte del profesorado, pues el hecho de seleccionar esta categoría y no la relativa a la falta de uso (No la he usado) implica cierto escepticismo en cuanto a la aplicabilidad de la técnica en la materia donde el profesor imparte docencia.

Seguidamente la Figura 1 muestra la representación bidimensional obtenida con análisis de correspondencias múltiples aplicado al análisis del uso. Como se observa en la figura, es posible distinguir tres grupos de profesores en cuanto al uso de dinámicas de trabajo en grupo. En primer lugar, aquellos que consideran que las técnicas de trabajo en grupo no se pueden aplicar en sus materias. En ellos se aprecia cierto distanciamiento entre el juego de rol y el resto de dinámicas, ya que al ser ésta la técnica con mayor porcentaje en la categoría No aplica, tiende a situarse más cercana al origen. Por el contrario, los casos de no aplicabilidad referidos a la pecera y los murmullos, en ambos casos con porcentajes de falta de aplicabilidad mínimos, se ubican en el extremo derecho de la representación, por tratarse de casos menos frecuentes. En segundo lugar, en el sector superior de la figura se aprecia, un segundo grupo de profesores que, en términos generales, utilizan las técnicas de trabajo en grupo. En este caso la posición más extrema la determinan tanto el uso de la bola de nieve como el de la pecera, ya que el uso de ambas técnicas es minoritario. Contrariamente, el puzzle, la tormenta de ideas y los dinamizadores se ubican más próximos al origen, al tener un uso más generalizado entre el profesorado universitario. En última instancia se encuentran los profesores que no hacen uso de estas técnicas, que por lo general constituyen la mayoría del colectivo, cercanos por tanto al origen.

Tabla 1: Porcentajes de uso de dinámicas de trabajo en grupo

\begin{tabular}{|l|ccc|}
\hline & Uso & No uso & No aplica \\
\hline BS & 50,0 & 45,2 & 4,8 \\
PZ & 45,0 & 51,3 & 3,7 \\
DN & 41,0 & 55,2 & 3,8 \\
MM & 31,8 & 66,5 & 1,7 \\
\hline JR & 20,2 & 54,4 & 25,4 \\
GN & 10,9 & 79,3 & 9,8 \\
\hline VM & 8,1 & 83,8 & 8,1 \\
BN & 7,4 & 86,7 & 5,9 \\
PC & 3,2 & 88,3 & 8,5 \\
\hline
\end{tabular}




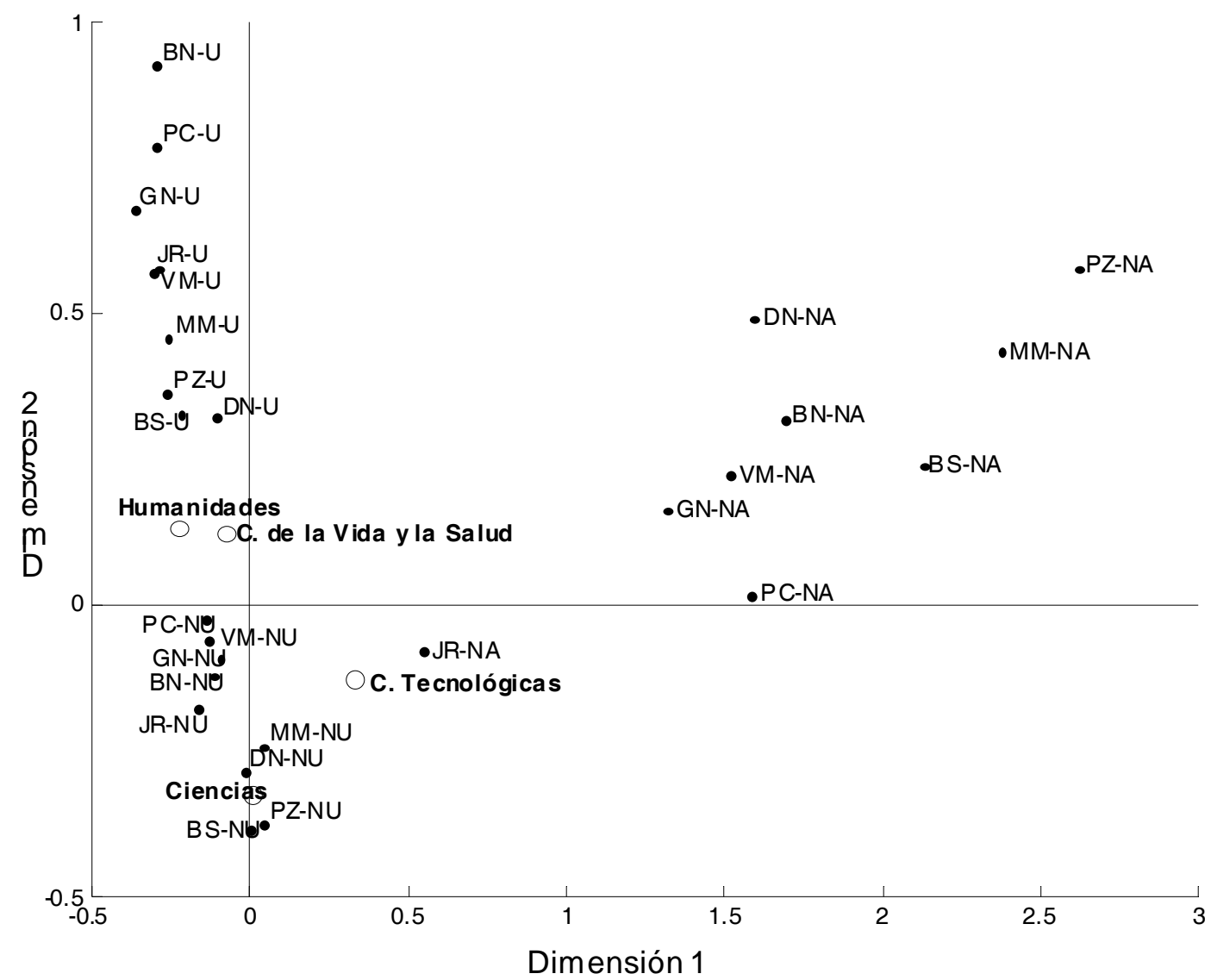

Fig. 1: Representación bidimensional del uso de las dinámicas de trabajo en grupo

Adicionalmente se ha proyectado como elemento suplementario el área de conocimiento del departamento donde cada profesor imparte la docencia. Este tipo de proyección se realiza con posterioridad a la obtención de los factores mostrados en la representación bidimensional. Por consiguiente, el área de estudio no interviene en el cálculo de los factores, aunque sí es posible analizar su posición respecto a dichos ejes. Así, en la Figura 1 se observa cómo las Ciencias Tecnológicas tienden a ubicarse hacia la zona de no aplicabilidad, señalando la asociación entre esta área de estudio y cierto rechazo hacia la posible aplicación de las dinámicas de trabajo en grupo en este campo. Asimismo, las áreas de Humanidades y Ciencias de la Vida y la Salud, se sitúan cerca del origen aunque en el sector superior del plano, indicando cierta relación con el uso generalizado de las dinámicas de trabajo en grupo. Por último, el área de Ciencias se ubica en el sector inferior del plano, vinculado a la falta de uso de estas técnicas pero no mostrando una oposición al posible uso de las mismas.

Independientemente del área de conocimiento, el perfil profesional del profesorado muestra en ocasiones una influencia considerable en el uso de las dinámicas de trabajo en grupo. Concretamente, la experiencia docente, medida mediante el número de años en docencia universitaria, resulta un factor determinante. En este aspecto, los profesores que imparten docencia en una franja comprendida entre 6 y 11 años de clase, son los que más uso hacen de las dinámicas de trabajo en grupo. Los resultados de la Tabla 2 muestran que el porcentaje de profesores con mayor o menor antigüedad a esta franja hacen un uso más limitado de estas técnicas. Igualmente el hecho de ser el responsable de la asignatura repercute en un mayor uso de las técnicas de trabajo en grupo.

Por otro lado, tanto la percepción de los conocimientos que se poseen como la opinión sobre las metodologías activas, guardan relación con su empleo. Específicamente, hacen mayor uso aquellos profesores que afirman tener conocimientos moderados frente a los que manifiestan tener conocimientos a nivel de principiante. Del mismo modo, el uso de estas técnicas es superior entre aquellos que tienen una opinión favorable o muy favorable hacia las metodologías activas. Al mismo tiempo, cabe señalar la relación entre los conocimientos y la opinión sobre estas 
metodologías. Como cabría esperar, el porcentaje de profesores con conocimientos moderados es superior entre aquellos que tienen una opinión favorable sobre metodologías activas (29.2\%) o muy favorable (58.3\%), frente a aquellos que se manifiestan neutrales (12.5\%) o en contra (0.0\%). En cuanto al área de conocimiento, la Tabla 2 confirma los resultados mostrados en la Figura 1, según los cuales los profesores que imparten docencia en las áreas de Humanidades y Ciencias de la Vida y la Salud hacen mayor uso de estas técnicas, en contraposición a las áreas de Ciencias y Ciencias Tecnológicas.

Sin embargo, la falta de uso no implica rechazo a su aplicabilidad, sino que puede deberse a otras causas más directas, como por ejemplo el desconocimiento o la falta de formación sobre su empleo en el aula. Este aspecto puede estudiarse mediante el análisis de los porcentajes de profesores que seleccionaron la categoría No aplica. Como se observa en la Tabla 3, estos porcentajes son superiores en Ciencias Tecnológicas respecto a los obtenidos en Ciencias, donde sólo alcanzan porcentajes elevados de falta de aplicabilidad el juego de rol y el voto múltiple. Este resultado indica cierta tendencia generalizada en el área de Ciencias Tecnológicas hacia el rechazo de la aplicabilidad de estas técnicas. Por el contrario en el área de Ciencias no se da esta tendencia, a pesar de que los porcentajes de uso mostrados en la Tabla 2 fueron tan reducidos como los obtenidos en Ciencias Tecnológicas. Por otro lado, en las áreas donde los profesores señalaron un uso más extendido de estas técnicas también se observan diferencias. Así, mientras que en el área de Ciencias de la Vida y la Salud los porcentajes de falta de aplicabilidad son relativamente moderados, en el área de Humanidades dichos porcentajes son prácticamente nulos, poniendo de manifiesto la predisposición del profesorado hacia la integración de estas dinámicas como parte de las metodologías docentes.

Tabla 2: Porcentajes de uso de dinámicas de trabajo en grupo, por variables del perfil del profesorado (**Nivel de significación $\alpha=5 \%$; *Nivel de significación $\alpha=10 \%$ ).

\begin{tabular}{|c|c|c|c|c|c|c|c|c|c|c|}
\hline & & $J R$ & PC & PZ & DN & GN & BS & VM & MM & $\mathrm{BN}$ \\
\hline \multirow{3}{*}{$\begin{array}{l}\text { Años de } \\
\text { docencia } \\
\text { universitaria }\end{array}$} & $<=5$ & 16,4 & 1,6 & $36,9 * \star$ & 35,4 & $4,8^{*}$ & $46,2^{\star \star}$ & 6,2 & 30,0 & 3,0 ** \\
\hline & 06 - 11 años & 29,7 & 8,1 & $72,2^{\star \star}$ & 38,2 & $18,2^{*}$ & $68,6^{* *}$ & 13,9 & 38,9 & $19,4^{\star *}$ \\
\hline & $>11$ & 17,2 & 1,8 & $40,4^{\star \star}$ & 42,6 & $12,3^{*}$ & $44,8^{\star *}$ & 9,3 & 32,7 & $5,3^{* *}$ \\
\hline \multirow{2}{*}{$\begin{array}{l}\text { Responsable } \\
\text { de la } \\
\text { asignatura }\end{array}$} & Sí & 20,9 & 3,7 & $53,3^{\star *}$ & $44,2^{\star \star}$ & $14,3^{*}$ & $57,5^{\star *}$ & 10,6 & $36,9^{*}$ & $9,3^{\star}$ \\
\hline & No & 18,2 & 3,1 & $35,4^{\star \star}$ & $30,2^{\star \star}$ & $6,3^{*}$ & 40,0 ** & 6,2 & $24,2^{*}$ & $3,1^{*}$ \\
\hline \multirow{2}{*}{$\begin{array}{l}\text { Conocimientos } \\
\text { o experiencia } \\
\text { en DTG }\end{array}$} & Principia & $14,5^{\star \star}$ & 2,7 & 39,9 ** & 35,4 & $6,9^{\star \star}$ & 47,3 & $4,8^{\star \star}$ & $27,9^{*}$ & 4,1 \\
\hline & Moderados & $44,8^{\star *}$ & 6,9 & 69,0 ** & $57,1^{\star *}$ & $28,6^{\star *}$ & 62,1 & 25,0 ** & $44,8^{*}$ & 24,1 \\
\hline \multirow{4}{*}{$\begin{array}{l}\text { Opinión sobre } \\
\text { Metodologías } \\
\text { activas }\end{array}$} & En con & 25,0 & 12,5 & 25,0 ** & $28,6^{*}$ & 0,0 & 37,5 & 0,0 & 12,5 & 0,0 \\
\hline & Depe & 6,5 & 3,2 & $33,3^{\star *}$ & $26,7^{*}$ & 16,1 & 38,7 & 6,5 & 21,4 & 6,7 \\
\hline & $\begin{array}{l}\text { Favorable } \\
\text { Muy }\end{array}$ & 21,5 & 3,2 & $43,8^{\star \star}$ & $33,9^{*}$ & 8,3 & 55,7 & 13,1 & 23,7 & 4,8 \\
\hline & & 32,6 & 2,2 & 62,2 & $55,8^{*}$ & 18, & 58,7 & 4,5 & 40 & 10,9 \\
\hline \multirow{4}{*}{$\begin{array}{l}\text { Área de } \\
\text { conocimiento }\end{array}$} & $\begin{array}{l}\text { Ciencias } \\
\text { C. de la Vida }\end{array}$ & $0,0^{\star \star}$ & 0,0 & $27,3^{\star \star}$ & 28,6 & $0,0^{\star \star}$ & 27,3 & 4,8 & $27,3^{*}$ & 0,0 \\
\hline & $\begin{array}{l}\text { y la Salud } \\
\text { C. }\end{array}$ & $29,3^{* *}$ & 5,0 & $57,5^{\star \star}$ & 38,5 & $23,7^{\text {** }}$ & 53,7 & 10,3 & $33,3^{*}$ & 9,8 \\
\hline & Tecnológ & $5,1^{\star \star}$ & 0,0 & $33,3^{\star *}$ & 31,6 & $5,3^{\star \star}$ & 36,8 & 8,1 & $18,9^{*}$ & 2,6 \\
\hline & Humanidades & $33,3^{\star \star}$ & 5,6 & $53,7^{\star \star}$ & 48,1 & $9,4^{\star \star}$ & 68,5 & 10,9 & $44,9 *$ & 11,3 \\
\hline
\end{tabular}

De la misma forma, existen ciertos factores del perfil del profesorado que pueden condicionar esta actitud de rechazo hacia la aplicabilidad de estas técnicas en el aula. Concretamente los factores más relevantes son tener poca experiencia en docencia universitaria (aproximadamente menos de 6 años), tener conocimientos a nivel de principiante en estas metodologías y obviamente, mostrar cierta predisposición a su rechazo, que se manifiesta mediante una opinión en contra o no definida (depende) sobre las metodologías activas. 
Tabla 3: Porcentajes de falta de aplicabilidad de dinámicas de trabajo en grupo, por variables del perfil del profesorado

\begin{tabular}{|c|c|c|c|c|c|c|c|c|c|c|}
\hline & & $\mathrm{JR}$ & $\mathrm{PC}$ & $\mathrm{PZ}$ & DN & GN & BS & VM & MM & $\mathrm{BN}$ \\
\hline \multirow{2}{*}{$\begin{array}{l}\text { Años de } \\
\text { docencia } \\
\text { universitaria }\end{array}$} & $<=5$ & 49,0 & 13,2 & 5,9 & 13,4 & 26,3 & 10,3 & 18,5 & 1,7 & 11,7 \\
\hline & $>6$ & 22,4 & 8,9 & 1,8 & 1,9 & 7,0 & 6,9 & 7,4 & 1,8 & 5,3 \\
\hline \multirow{2}{*}{$\begin{array}{l}\text { Conocimientos o } \\
\text { experiencia en } \\
\text { DTG }\end{array}$} & Principiante & 27,6 & 10,2 & 4,1 & 4,2 & 9,7 & 6,1 & 7,5 & 1,4 & 6,1 \\
\hline & Moderados & 17,2 & 0,0 & 0,0 & 3,6 & 14,3 & 0,0 & 10,7 & 0,0 & 3,4 \\
\hline \multirow{2}{*}{$\begin{array}{l}\text { Opinión sobre } \\
\text { DTG }\end{array}$} & $\begin{array}{l}\text { En contra o } \\
\text { Depende }\end{array}$ & 60,5 & 25,4 & 3,3 & 6,7 & 25,4 & 19,0 & 12,9 & 0,0 & 6,7 \\
\hline & $\begin{array}{l}\text { Favorable o } \\
\text { Muy Favorable }\end{array}$ & 41,4 & 12,4 & 6,9 & 4,8 & 13,0 & 3,3 & 11,1 & 1,7 & 10,3 \\
\hline \multirow{4}{*}{$\begin{array}{l}\text { Área de estudio } \\
\text { del departamento }\end{array}$} & Ciencias & 40,9 & 4,8 & 0,0 & 0,0 & 9,5 & 4,5 & 14,3 & 0,0 & 0,0 \\
\hline & $\begin{array}{l}\text { C. de la Vida y } \\
\text { la Salud }\end{array}$ & 19,5 & 5,0 & 2,5 & 7,7 & 10,5 & 4,9 & 7,7 & 0,0 & 2,4 \\
\hline & C. Tecnológicas & 41,0 & 20,5 & 7,7 & 5,3 & 18,4 & 15,8 & 10,8 & 2,7 & 20,5 \\
\hline & Humanidades & 8,8 & 1,9 & 0,0 & 1,9 & 5,7 & 0,0 & 3,6 & 2,0 & 0,0 \\
\hline
\end{tabular}

Seguidamente se analizan las preferencias por las distintas dinámicas de trabajo en grupo, entre aquellos profesores que manifestaron utilizarlas. La Tabla 4 muestra los porcentajes correspondientes a las categorías La he usado y no me gusta y La he usado y me gusta etiquetadas como Uso y No gusta (UNG) y Uso y Gusta (UG), respectivamente. Como se observa en la tabla, los porcentajes de profesores que han usado y les gusta cada una de estas técnicas son siempre superiores a los que las han usado y no les gusta. Este resultado indica que sólo una minoría opta por no seguir utilizando las dinámicas de trabajo en grupo, después de haberlas empleado al menos una vez.

Puesto que los porcentajes de Uso y no gusta son muy reducidos, y en determinadas técnicas de trabajo en grupo, el número de casos es insuficiente para realizar análisis estadísticos, se han seleccionado las 4 dinámicas de uso mayoritario (tormenta de ideas, dinamizadores, puzzle y murmullos) para estudiar en profundidad las preferencias de uso del profesorado universitario de estas técnicas en concreto.

Tabla 4: Porcentajes de preferencias de uso de dinámicas de trabajo en grupo

\begin{tabular}{|l|cc|c|}
\hline & Uso y No gusta & Uso y Gusta & Total Uso \\
\hline BS & 3,2 & 46,8 & 50,0 \\
PZ & 2,1 & 42,9 & 45,0 \\
DN & 1,6 & 39,3 & 41,0 \\
MM & 5,0 & 26,8 & 31,8 \\
\hline JR & 1,0 & 19,2 & 20,2 \\
GN & 1,1 & 9,8 & 10,9 \\
\hline VM & 1,6 & 6,5 & 8,1 \\
BN & 0,5 & 6,9 & 7,4 \\
PC & 1,1 & 2,1 & 3,2 \\
\hline
\end{tabular}

El mapa de correspondencias mostrado en la Figura 2 permite identificar 4 perfiles de profesores, relacionados con los identificados en el análisis del uso, y mostrados en la Figura 1. Por un lado en el sector derecho se ubican las categorías de falta de aplicabilidad, como un grupo claramente diferenciado del resto, mientras que la falta de uso se sitúa en el sector inferior del grupo. Junto a estas categorías de falta de uso se encuentra también el Uso y No gusta, específico de los murmullos. Este resultado indica que existe un segundo perfil de profesores universitarios, que en general no hacen uso de las dinámicas de trabajo en grupo, y entre los cuales hay un porcentaje considerable que han usado la técnica de murmullos y no les ha gustado. Por el contrario, en el sector superior izquierdo se sitúan las categorías de Uso y Gusta, poniendo de manifiesto la existencia de un tercer perfil de profesores que en algún momento han empleado estas técnicas y 
les han gustado. Por último, cercanos al origen se sitúan las categorías Uso y No gusta, relativas a la tormenta de ideas, el puzzle y los dinamizadores. Estas categorías no se sitúan con claridad en ninguna posición diferenciada en el plano de correspondencias, ya que no se asocian directamente con los distintos perfiles identificados anteriormente.

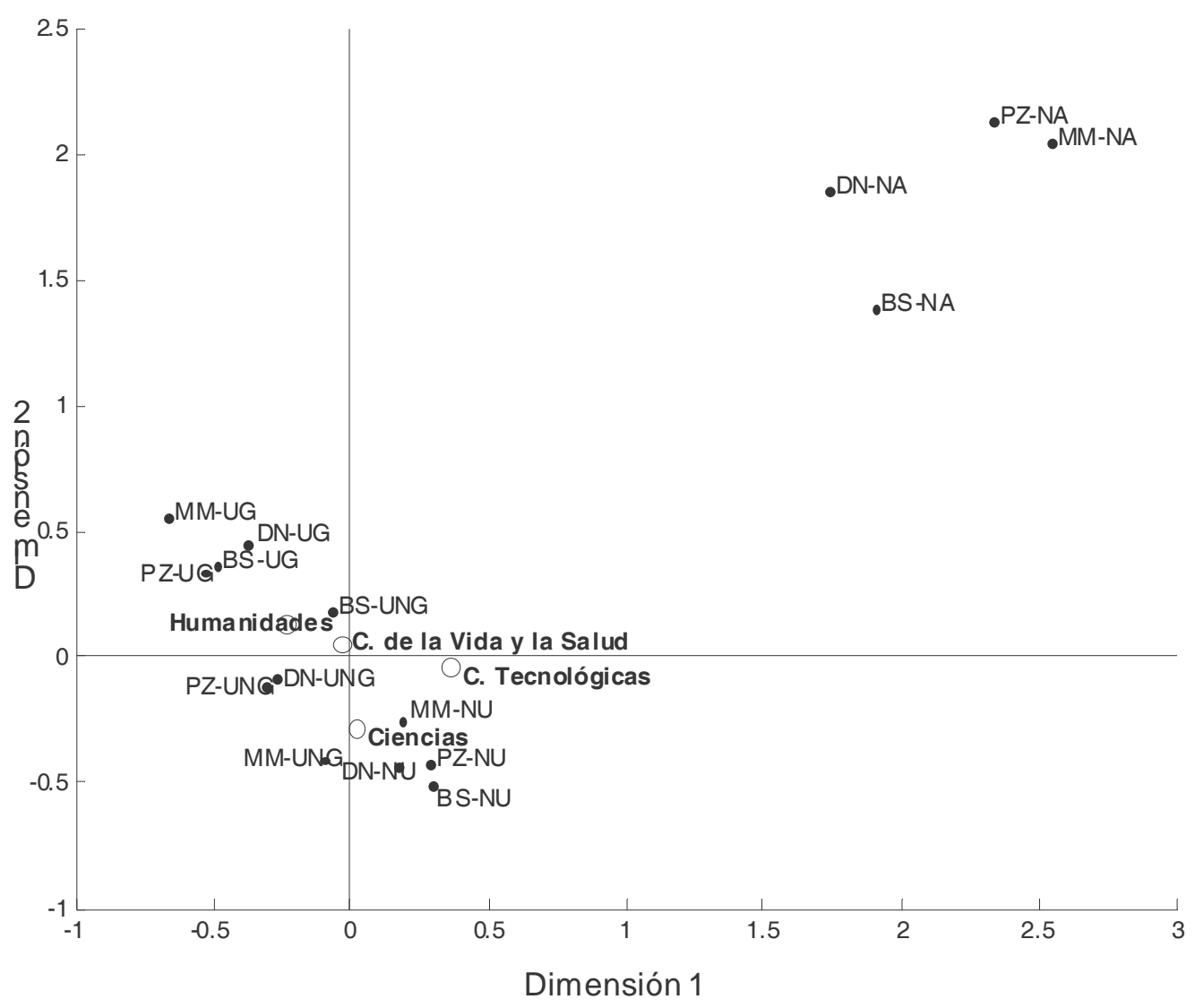

Fig. 2: Representación bidimensional de las preferencias de uso de las dinámicas de trabajo en grupo de uso mayoritario

Al igual que antes, se ha proyectado en suplementario la información sobre el área de estudio donde los profesores imparten la docencia. Las posiciones que ocupan en esta figura, son muy similares a las que se observaron en la Figura 1. En primer lugar las Ciencias Tecnológicas muestran cierta aproximación a las categorías de falta de aplicabilidad, mientras que las Ciencias se sitúan cerca de las categorías de falta de uso. Las Humanidades y Ciencias de la Vida y la Salud se ubican claramente entre las categorías de uso. Sin embargo, las Humanidades se encuentran ligeramente desplazadas hacia el sector izquierdo, mostrando mayor asociación con las categorías de Uso y Gusta.

En cuanto al perfil del profesorado y su relación con las preferencias de uso, se observa en la Tabla 5 cómo las variables identificadas anteriormente como relevantes en la determinación del uso, siguen siendo significativas respecto al uso y gusto por las técnicas de trabajo en grupo. Evidentemente esta relación está vinculada con el resultado comentado en la Tabla 4, donde se observó que sólo un pequeño porcentaje de los profesores que alguna vez habían usado estas técnicas, finalmente no les habían gustado. De este modo, parece tener cierta relación con la preferencia de uso por estas técnicas, el hecho de tener una experiencia en docencia universitaria comprendida en la franja entre 6 y 11 años, e igualmente ser el responsable de la asignatura donde se imparte la docencia. Por otro lado, continúa siendo determinante tener conocimientos moderados sobre estas dinámicas así como tener una opinión favorable o muy favorable sobre metodologías activas. En lo que respecta al área de conocimiento, se observa con facilidad que los porcentajes de preferencias de uso son siempre superiores en Humanidades, independientemente de la técnica considerada. 
Nuestra comunicación presenta varias aportaciones para investigadores en docencia, profesores y gestores de universidad. En primer lugar hace una síntesis de las principales actividades de grupo que pueden utilizarse en las aulas de grado. En segundo lugar, estima las variables explicativas del grado de uso y su importancia relativa a la hora de predecir el uso de las técnicas populares. Por último, permite identificar que el grado de uso depende tanto de aspectos culturales (posición frente a las metodologías activas y conocimientos sobre técnicas de grupo), como de aspectos estructurales (cantidad de profesores implicados en la docencia y tipo de profesor). De modo que se pueden articular las medidas más adecuadas para fomentar el uso de dinámicas de grupo en educación superior, en el caso de considerarse una metodología adecuada. Por ejemplo, formación para concienciar a los profesores (aspecto cultural) o dotar de recursos adecuados para la implantación de los nuevos grados (aspecto estructural).

Nuestro trabajo no está exento de limitaciones, la principal de ellas es que la muestra utilizada es de conveniencia y no nos permite generalizar las conclusiones a todo el contexto universitario español salvo que el perfil de asistentes a los talleres pudiera considerase representativo de un colectivo importante de profesores del sistema universitario público.

Como líneas de trabajo futuras nos planteamos el incorporar el tipo de contenidos de la asignatura o el curso en que se imparte, como variables explicativa del grado de uso de técnicas de grupo. También nos planteamos realizar un análisis cualitativo de ventajas e inconvenientes que perciben los profesores de cada una de las técnicas y la relación que guardan estas percepciones con el grado de uso de las técnicas de trabajo en grupo en las aulas universitarias.

Tabla 5: Porcentajes de uso y gusto de dinámicas de trabajo en grupo, por variables del perfil del profesorado (**Nivel de significación $\alpha=5 \%$; *Nivel de significación $\alpha=10 \%$ ).

\begin{tabular}{|c|c|c|c|c|c|}
\hline & & $P Z$ & DN & BS & MM \\
\hline \multirow{3}{*}{$\begin{array}{l}\text { Años de docencia } \\
\text { universitaria }\end{array}$} & $<=5$ & $35,4^{\star \star}$ & 33,80 & $44,6^{\star \star}$ & 25,00 \\
\hline & 06 - 11 años & $69,4^{\star \star}$ & 38,20 & 62,9 ** & 33,30 \\
\hline & $>11$ & $38,6^{\star \star}$ & 40,70 & $39,7^{\star *}$ & 27,30 \\
\hline \multirow{2}{*}{$\begin{array}{l}\text { Responsable de la } \\
\text { asignatura }\end{array}$} & Sí & $51,4^{\star \star}$ & $42,3^{\star \star}$ & $53,8^{\star \star}$ & $32,0^{*}$ \\
\hline & No & $33,8^{* *}$ & $28,6^{\star \star}$ & $36,9^{\star \star}$ & $21,0^{*}$ \\
\hline \multirow{2}{*}{$\begin{array}{l}\text { Conocimientos o } \\
\text { experiencia en DTG }\end{array}$} & Principiante & $37,8^{\star *}$ & 34,0 ** & 44,60 & $22,9^{* \star}$ \\
\hline & Moderados & 69,0 ** & $57,1^{\star *}$ & 55,20 & $41,4^{\star *}$ \\
\hline \multirow{4}{*}{$\begin{array}{l}\text { Opinión sobre } \\
\text { Metodologías activas }\end{array}$} & En contra & $12,5^{\star \star}$ & 28,60 & 37,50 & $12,5^{\star \star}$ \\
\hline & Depende & 30,0 ** & 26,70 & 38,70 & $14,3^{* \star}$ \\
\hline & Favorable & $43,8^{\star *}$ & 32,30 & 50,80 & $18,6^{\star *}$ \\
\hline & Muy Favorable & 60,0 ** & 51,20 & 52,20 & $36,4^{\star *}$ \\
\hline \multirow{4}{*}{$\begin{array}{l}\text { Área de } \\
\text { conocimiento }\end{array}$} & Ciencias & $27,3^{\star *}$ & 28,60 & $27,3^{\star \star}$ & $22,7^{*}$ \\
\hline & C. de la Vida y la Salud & $57,5^{\star \star}$ & 35,90 & $51,2^{\star \star}$ & $25,6^{*}$ \\
\hline & C. Tecnológicas & $28,2^{* *}$ & 28,90 & $31,6^{* *}$ & $16,2^{*}$ \\
\hline & Humanidades & 51,9 ** & 46,20 & $63,0 * *$ & $38,8^{*}$ \\
\hline
\end{tabular}

\section{CONCLUSIONES}

La presente investigación ha permitido identificar la tormenta de ideas, el puzzle, los dinamizadores y los murmullos como las dinámicas de trabajo en grupo más utilizadas por los profesores universitarios. En contraposición, el voto múltiple, la bola de nieve y la pecera se muestran como técnicas de uso minoritario. En término medio se encuentran el juego de rol y los grupos nominales, aunque encabezan el ranking de las más rechazadas en cuanto a su aplicabilidad en el aula.

Respecto al uso de las técnicas de trabajo en grupo, se han identificado mediante análisis de correspondencias múltiple tres perfiles de profesores universitarios. En primer lugar aquellos que rechazan su posible aplicación en las materias donde imparten docencia. Ser profesor del área de Ciencias Tecnológicas, así como tener poca experiencia en docencia universitaria (menos de 5 
años), conocimientos a nivel de principiante sobre esta cuestión o mostrar una predisposición a su rechazo a través de una opinión en contra o indefinida hacia las metodologías activas, son las características predominantes de los profesores incluidos en este primer grupo. Por el contrario existe un segundo grupo de profesores que sí utilizan estas técnicas en el aula. En este segundo grupo es fácil encontrar a profesores del área de Humanidades y Ciencias de la Vida y la Salud, así como con una experiencia docente comprendida entre 6 y 11 años, habitualmente responsables de asignatura, con conocimientos moderados sobre estas técnicas y opiniones favorables o muy favorables sobre metodologías activas. Por último existe un tercer grupo de profesores que habitualmente no utilizan estas técnicas. Entre ellos se encuentra un considerable porcentaje de profesores de Ciencias, así como profesores con poca o mucha experiencia en docencia universitaria (menos de 5 años o más de 11).

Por otro lado se ha destacado el hecho de que la gran mayoría de los profesores que alguna vez han empleado alguna de estas técnicas han manifestado que les ha gustado. Específicamente sobre la preferencia de uso se han identificado dos grupos, adicionales a los identificados anteriormente. Los profesores clasificados en el primero de ellos señalaron que les había gustado usar estas técnicas en el aula, mientras que el segundo de ellos afirma lo contrario. No obstante, este último grupo no toma una posición claramente diferenciada en el plano de correspondencias. Únicamente destaca el caso de los murmullos, donde la categoría Uso y no gusta se sitúa cercana al grupo de falta de uso, señalando que un porcentaje considerable de profesores que habitualmente no usan estas técnicas, manifestaron que no les gustaron los murmullos después de haberlas empleado en clase.

\section{AGRADECIMIENTOS}

Este artículo ha contado con la financiación del proyecto "Construcción de un cuestionario de factores psicosociales para el diagnóstico de condiciones de trabajo y efectos sobre la salud y la satisfacción laboral. Adaptación a entornos docentes universitarios" (PAID-05-11-2846) de la Universitat Politècnica de València y el proyecto PAID-08-10 también de la Universitat Politècnica de València

\section{REFERENCIAS}

Anson, C. M.; L. E. Bernold; C. Crossland; J. Spurlin; M. A. McDermotr y S. Weiss; Empowerment to Learn in Engineering: Preparation Foran Urgently-Needed Paradigm Shift, Global Journal of Engineering Education: 7(2), 145-155 (2003).

Auster, E. R. y K. K. Wylie; Creating Active Learning in the Classroom: A Systematic Approach, Journal of Management Education: 30(2), 333-353 (2006).

Brewer, W. y M. I. Mendelson; Methodology and Metrics for Assessing Team Effectiveness, International Journal of Engineering Education: 19(6), 777-787 (2003).

Contreras Palma, S. A.; Qué Piensan Los Profesores Sobre Sus Clases: Estudio Sobre Las Creencias Curriculares y Las Creencias De Actuación Curricular, Formación Universitaria: 1(3), 3-11 (2008).

Fabra, M. L.; Técnicas de grupo para la cooperación, CEAC, Barcelona (1994).

Fruchter, R.; Dimensions of Teamwork Education, International Journal of Engineering Education: 17(45), 426-430 (2001).

Greenacre, M.; La práctica del análisis de correspondencias, Fundación BBVA, www.fbbva.es (2008).

Holtham, C. W.; R. R. Melville y M. S. Sodhi; Designing Student Groupwork in Management Education: Widening the Palette of Options, Journal of Management Education: 30(6), 809-817 (2006).

Jenkins, H. y L. W. Lackey; Preparing Engineering Students for Working in Teams Through Senior Design Projects, IEEE International Professional Communication Conference Proceedings., (2005). 
Kalliath, T. y M. Laiken; Use of Teams in Management Education, Journal of Management Education: 30(6), 747-750 (2006).

Lebart, L.; Morineau, A. y Piron, M.; Statistique Exploratoire Multidimensionnelle, Dunod, Paris (1995).

Lizasoain, L. y L. Joaristi; Las Nuevas Tecnologías y La Investigación Educativa. El Análisis De Datos De Variables Categoriales, Revista Española De Pedagogía:(251), 111-130 (2012).

Lloret, J. y J. A. Marin-Garcia; Novel and Stable Lecturers' Point of View About University Students Working Groups, in Mathematical Methods and Computational Techniques in Research and Education by Dondon, P. et al., pp 368-373 WSEAS Press, Arcachon, France (2007).

Lloret, J. y J. A. Marin-Garcia; Comparing Novel and Stable Lecturers' Point of View When They Use University Students Working Groups in Their Classrooms, WSEAS Transactions on Advances in Engineering Education: 11(5), 699-708 (2008).

Marin-Garcia, J. A.; J. P. Garcia-Sabater; M. R. Perello-Marin y L. Canos-Daros; Proposal of Skills for the Bachelor Degree of Industrial Engineering in the Context of the New Curriculum, Intangible Capital: 5(4), 387-406 (2009).

Marin-Garcia, J. A. y J. Lloret; Improving Teamwork With University Engineering Students. The Effect of an Assessment Method to Prevent Shirking, WSEAS Transactions on Advances in Engineering Education: 5(1), 1-11 (2008).

Marin-Garcia, J. A.; T. Marin-Garcia; M. R. Perello-Marin y J. J. Garcia-Sabater; Selección De Plataformas Para El Trabajo Colaborativo En Grupos Deslocalizados: Formulación Del Problema, Working Papers on Operations Management: 1(1), 41-45 (2010).

Marin-Garcia, J. A.; C. Miralles Insa; J. J. Garcia-Sabater y P. I. Vidal Carreas; Teaching Management Based on Students Teamwork: Advantages, Drawbacks and Proposals for Action, Intangible Capital: 4(2), 143-165 (2008).

Martínez Gómez, M. y J. A. Marin-Garcia; Cómo Medir y Guiar Cambios Hacia Entornos Educativos Más Motivadores, Formación Universitaria: 2(4), 3-14 (2009).

Michaelson, R., (2003). Assessing Group Work. Briefing paper for LTSN-BEST. http://www.business.heacademy.ac.uk/publications/misc/briefing/groupwork/assessing\%20group\%20w ork\%20-\%20michaelson.pdf. Last accesed april 2007.

O'Doherty, D. M.; Working As Part of a Balanced Team, International Journal of Engineering Education: 21(1), 113-120 (2005).

Palou, M. y J. Montaño; Análisis Del Trabajo Presencial y No Presencial De Profesores y Alumnos Bajo El Concepto Del Sistema De Transferencia Europeo De Créditos (ECTS), Formación Universitaria: 1(4), 3-12 (2008).

Raposo Rivas, M. y M. E. Martínez Figueira; La Rúbrica En La Enseñanza Universitaria: Un Recurso Para La Tutoría De Grupos De Estudiantes, Formación Universitaria: 4(4), 19-28 (2011).

Sheppard, K.; P. Dominick y Z. Aronson; Preparing Engineering Students for the New Business Paradigm of International Teamwork and Global Orientation, International Journal of Engineering Education: 20(3), 475-483 (2004).

Watts, F.; A. García-Carbonell y J. Llorens; Introducción a La Evaluación Compartida: Investigación Multidisciplinar, in La Evaluación Compartida: Investigación Multidisciplinar by Watts, F. \& GarcíaCarbonell, A., pp 1-9 Editorial de la UPV, Valencia (2006).

Young, C. B. y J. A. Henquinet; A Conceptual Framework for Designing Group Projects., Journal of Education for Business: 76(1), 56-60 (2000). 